\title{
The scope of public health research strategies
}

\author{
Dörte Pippel
}

Published online: 30 October 2012

(C) Springer-Verlag Berlin Heidelberg 2012

Major health problems facing the world today include infectious diseases, chronic diseases, trauma, and mental health. Looking back unto the nineteenth and early twentieth centuries, people primarily faced infectious diseases like tuberculosis, enteric infections and acute respiratory diseases due to undernutrition, low hygiene and bad working conditions during the early industrialization. These problems still persist in many parts of the world; however, the beginning of the twenty-first century has come up with another set of health problems which result from modern lifestyle choices like an overly rich or unbalanced diet, tobacco and drug use, alcohol consumption, too little physical activity and other conditions that shape the modern way of life of people in developed and highly industrialized countries. Coronary heart diseases, cancer, other chronic diseases and also mental disorders are predominant in most developed nations and are coming to the fore in developing countries (Hamlin 2004).

Public health tries to cope with such a variety of health problems by including scientific strategies from the epidemiological as well as from the social, biological and physical sciences. This interdisciplinary approach results from the knowledge that health problems today are provoked by a variety of different determinants of health which include nutritional problems, social and environmental hazards and disorders resulting from lifestyle choices. Public health measures for preventing diseases and for influencing the health of communities and populations face the challenge of considering all these various (modern) determinants of health. Against this background, public health research

\section{Pippel $(\bowtie)$}

Research Association Public Health Saxony, Faculty of Medicine,

TU Dresden,

Fiedlerstr. 33,

01307 Dresden, Germany

e-mail: doerte.pippel@mailbox.tu-dresden.de focuses its activities on four major strategies for influencing health - preventing disease and promoting health, improving medical care, promoting health-enhancing behavior, and controlling the environment (Detels and Breslow 2004).

Taking into account the variety of health problems in a globalized world with populations facing different health determinants, the Journal of Public Health has responded to the current scopes and concerns of public health by displaying a wide range of research topics in an international arena. This issue of the Journal of Public Health includes articles from eight countries. Three articles focus public health issues in India; others attend to public health themes in Ireland, Italy, Greece, Finland, Uganda, USA, and Germany. It is just this consideration of internationality and interdisciplinary approaches as presented in the current articles that underlines the journal's aim to allow for a wide range within the different fields of public health research. The authors of today's edition present research topics on outcomes from lifestyle choices, research in improving medical care and social participation, findings in the treatment of infectious diseases as well as further knowledge in promoting health-enhancing behavior and health policy.

Habermannn et al. add to the development of sustainable risk management in patient care by presenting the findings of a cross-sectional study on the knowledge about nurses' error perception. Knowledge regarding categories and frequencies of errors identified by nurses is essential for the establishment of error-prevention strategies. Improving medical care is likewise an aim of the study on maternal healthcare service utilization in India introduced by Singh et al. The authors examine factors associated with the utilization of maternal healthcare services by married adolescent women within the ages of 15-19 years. Findings underline the importance of promoting health care and raising awareness about health problems from early childbearing among adolescent women. 
In Uganda, Mugambe et al. investigated the barriers that people living with HIV/AIDS (PLWHA) face in accessing water, sanitation and hygiene. The qualitative content analysis showed significant limitations in the access to improved water sources due to financial, attitudinal, social and institutional. Feelings of inferiority and isolation among the affected people were identified as worrying outcomes of this study.

Tiesler et al. links residential mobility during childhood and behavioural problems in school-age children. Their findings point to an increased risk for the development of behavioural problems among school-age children who have moved twice or more or who moved more than $50 \mathrm{~km}$ in total.

Mobility in the social context is associated with disorders resulting from lifestyle choices by the researchers Kuntz and Lampert. Using the data from the German KiGGS study, they investigate upward social mobility as a possible protective factor against obesity for children of lowereducated parents. According to the authors' conclusion, upward social mobility can be interpreted as a protective factor against obesity. Special lifestyle choices of laborers in Bhubaneswar, India are focused on in a cross-sectional study by Panigrahi et al. The study examines the prevalence of and questions underlying reasons for tobacco use among daily wage laborers. Feelings of pleasure but also peer pressure could be identified as primary motivations for smoking and chewing tobacco. The researchers stress the urgent need of intervention measures against tobacco use among labor classes in India.

A cross-sectional population study from Ireland, conducted by Barrett et al. examined the knowledge and practices of general practitioners and physiotherapists in the promotion of physical activity in primary care. They conclude that physical activity promotion needs further improvement especially among healthy populations. Underlining the need for promoting health is also the main aim of the study from Italy. In their study, La Torre et al. focused on the changes in knowledge and behaviors of Italian university students about sexual attitudes, use and effectiveness of contraceptive methods and sexually transmitted diseases (STD) by carrying out three surveys in 1998, 2003 and 2008. Results show that further promotion programs on sex education, the use of contraceptives and STD prevention are necessary among Italian adolescents.

In Finland, Liisanantti et al. undertook a study to examine the long-term mortality in patients hospitalized due to acute self-induced drug poisoning between 1985 and 2000 . Patients were then followed until the end of 2009 and compared to sex and year of birth matched controls. The results of the study indicate poor long-term outcomes of patients who had done acute self-poisoning.

Against the background of rapidly declining response rates in community surveys, an American research group led by R.S. Choung estimated the potential for response bias in standard mailed questionnaires used in surveys of gastroenterological symptoms. Eventually, non-response bias seems not to influence the results of a community-based GI survey in a major way.

The feasibility for injury prevention-related policy transfer among European countries was evaluated by a Greek research group. Under the instruction of Vlachantoni et al., Public health experts from Europe and the USA scored the transferability of a developed prototype for policy feasibility evaluation for the selected policies in their own country.

The third contribution from India gives an overview on the status of multidrug resistance in tubercle bacillus and phytochemicals for the control. Dubey et al. present in their study the possibility of the control of the appalling tubercle bacillus (TB) strains with pure phytochemicals as a complementary source of drugs. The authors conclude that pure phytochemicals should be considered in TB treatment these days.

In summary, the articles in this issue of the Journal of Public Health illustrate very well the broad spheres of action of the major strategies of public health for influencing health. The interdisciplinary and international scope of the Journal of Public Health allows the reader to gain insight into the current challenges and tasks of public health research today.

\section{References}

Detels R, Breslow L (2004) Current scope and concerns of public health. In: Detels R, McEwen J, Beaglehole R, Tanako H (eds) Oxford textbook of public health. Oxford University Press, New York, pp 3-21

Hamlin C (2004) The history and development of public health in developed countries. In: Detels R, McEwen J, Beaglehole R, Tanako H (eds) Oxford textbook of public health. Oxford University Press, New York, pp 21-39 